\title{
ALMA IM ÖSTERREICHISCHEN BIBLIOTHEKENVERBUND UND DIE PERSPEKTIVEN FÜR DIE ÖSTERREICHISCHE BIBLIOTHEKENVERBUND UND SERVICE GMBH. 10 FRAGEN VON BRUNO BAUER AN WOLFGANG HAMEDINGER, GESCHÄFTSFÜHRER DER OBVSG
}

\author{
von Bruno Bauer \& Wolfgang Hamedinger
}

Zusammenfassung: Seit 2017 erfolgt im Österreichischen Bibliothekenverbund eine Umstellung vom bisherigen Bibliotheksverwaltungssystem Aleph 500 auf das neue System Alma. Im Interview informiert Wolfgang Hamedinger, Geschäftsführer der Österreichischen Bibliothekenverbund und Service GmbH (OBVSG), über die besonderen Herausforderungen dieses Projektes, über dessen bisherige Umsetzung sowie über die geplanten weiteren Schritte. Angesprochen werden auch Themen wie die weiteren Geschäftsfelder der OBVSG und die Kooperation E-Medien Österreich, die Finanzierung der OBVSG sowie Charakteristika, die den Österreichischen Bibliothenverbund auszeichnen. Zuletzt beantwortet Hamedinger die Frage, ob es in zehn Jahren noch Bibliotheken und auch Bibliotheksverbünde geben wird.

Schlüsselwörter: Österreichischer Bibliothekenverbund; OBV; Österreichische Bibliothekenverbund und Service GmbH; OBVSG; Bibliothekssystemwechsel; Bibliotheksverwaltungssystem; Aleph 500; Alma; Geschäftsfelder; Kooperation E-Medien Österreich; KEMÖ; Finanzierung; Bibliotheken; Bibliotheksverbünde; Zukunft; Wolfgang Hamedinger; Interview

\section{ALMA IN THE AUSTRIAN LIBRARY NETWORK AND THE PERSPECTIVES FOR THE AUSTRIAN LIBRARY NETWORK AND SERVICE LTD: 10 QUESTIONS BY BRUNO BAUER TO WOLFGANG HAMEDINGER, MANAGING DIRECTOR OF THE OBVSG}

Abstract: Since 2017 the Austrian Library Network (ALN) is steadily replacing its previous integrated library system software Aleph 500 with the new unified resource management system ALMA. In the interview Wolfgang Hamedinger, director of the Austrian Library Network and Service Ltd, informs about the special challenges of this endeavour, its implementation so far and the planned next steps. Further topics like other business areas of the ALN and Austrian Academic Library Consortium, funding 
of ALN and characteristics that are distinguishing the Austrian Library Network are also discussed. In the end Hamedinger answers the question if there will be libraries and library networks in ten years time.

Keywords: Austrian Library Network; ALN; Austrian Joint Library System; library system change; integrated library system; Aleph 500; unified resource management; Alma; business areas; Austrian Academic Library Consortium; funding; libraries; library networks; future; Wolfgang Hamedinger; interview

DOI: https://doi.org/10.31263/voebm.v71i2.2138

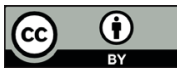

Dieses Werk ist lizenziert unter einer

Creative-Commons-Lizenz Namensnennung 4.0 International

\section{Systemwechsel von Aleph 500 auf Alma}

Bauer: Lieber Wolfgang, seit 25 Jahren - seit 1993, dem Jahr Deiner Bestellung zum Leiter der Planungsstelle für wissenschaftliches Bibliothekswesen - bist Du für die EDV-Entwicklung im Österreichischen Bibliothekenverbund verantwortlich. Bereits die Umstellung von BIBOS zu Aleph im Jahr 1999 hast Du an führender Stelle betrieben. Wenn Du den damaligen Systemwechsel mit der aktuellen Umstellung von Aleph zu Alma vergleichst: Inwieweit war der aktuelle Wechsel aufgrund der früheren Erfahrungen bereits Routine? Und was waren die besonderen Herausforderungen des aktuellen Systemwechsels?

Hamedinger: Lieber Bruno, das ist etwas viel der Ehre. Verantwortlich war und bin ich in dem Sinne, dass schon immer generelle Koordinationsaufgaben zu meinen Tätigkeiten gehörten und ich insbesondere seit Entstehung der OBVSG auch echte Entscheidungsbefugnisse habe. Aber selbst das reicht nicht für Alleingänge, sondern es war schlussendlich jedesmal ein mehr oder weniger breites Gemeinschaftsunternehmen; die Übernahme einer gewissen Koordinierungs-, Lenkungs- und manchmal auch Führungsrolle liegt nach unserem gesetzlichen Auftrag dann in der Natur der Sache.

Die Verwendung des Begriffs Routine wird - glaube ich - niemand mit der Umstellung Befasster in Erwägung ziehen. Ganz abgesehen davon, dass wir von einem zeitlichen Abstand von beinahe 20 Jahren zwischen den beiden Umstellungen sprechen, in denen wohl jede nicht laufend genutzte Fähigkeit eingerostet wäre, waren die jeweiligen Ausgangssituationen auch sehr unterschiedlich. 
Bei der Ablöse von BIBOS hatten wir einen Verbund, der im wesentlichen aus der Österreichischen Nationalbibliothek und den meisten Universitätsbibliotheken sowie wenigen anderen Einrichtungen bestand. Die Steuerungskompetenz in Form von Hoheit über die Finanzmittel lag (cum grano salis) beim Wissenschaftsministerium, die Unabhängigkeit der Universitäten gab es noch nicht. Funktionale Ausgangssituation und Komplexität waren weit weniger anspruchsvoll als beim momentan stattfindenden Systemwechsel; gerade wegen der Unzulänglichkeiten, kombiniert mit der Gefahr der Erreichung von Systemgrenzen und der sogenannten "Jahr-2000-Problematik" waren wir damals unter Umstellungsdruck. Man muss sich vergegenwärtigen, dass wir einen wahren Zoo von Systemen hatten: sieben, die von mehreren Einrichtungen genutzt wurden und drei weitere als lokale Eigenentwicklungen. Jedes davon beherrschte einen Teil der nötigen Funktionalitäten mehr oder weniger und wir waren dauernd damit beschäftigt, jedes dieser einzelnen Systeme passend mit Daten aus anderen zu versorgen und dieses Sammelsurium konsistent zu halten. So unterschiedlich wie die Systeme war dann auch die jeweils verwendete Technologie, vom Großrechner über DOSUmgebungen bis zu proprietären anderen Betriebssystemen und Hardware. Die heute beherrschenden Linux/Unix-Systeme waren in unserem Bereich noch nicht verbreitet und kamen nur in einem Fall zum Einsatz. Diese nur lose gekoppelten Systeme erlaubten uns dafür allerdings, die Migration für alle Anwender des allgemeinen Katalogisierungssystems BIBOS-2 nach Aleph in einem großen Schritt durchzuführen. Die anderen Systeme konnten dann schrittweise durch Aktivierung der entsprechenden Funktionalitäten in Aleph 500 entsprechend den lokalen Möglichkeiten abgelöst werden.

In den fast zwei Jahrzehnten des Einsatzes von Aleph als integriertes Bibliothekssystem sind die Möglichkeiten des Systems ausgereizt und einige Dienste in die Gesamtarchitektur integriert worden. Der Verbund ist wegen seiner Vorteile in dieser Zeit stark gewachsen und trotz bedeutender Organisationsänderungen, wie der Ausgliederung der Verbundzentrale als $\mathrm{GmbH}$ und der Verselbständigung der Österreichischen Nationalbibliothek und der Universitäten ein Modellfall der Zusammenarbeit geblieben. Ein Übergang zu einem neuen System bringt hier ganz neue Herausforderungen. Zuallererst wohl die Einsicht, dass man diesen Übergang überhaupt einleiten muss, obwohl das alte System sehr gut funktioniert und alles eingespielt ist und besonders heikel, die Frage des geeigneten Zeitpunkts. Wie man so ein Projekt angehen kann und finanziert. Wie man von der alten Systemumgebung in die neue kommt, ohne in der Übergangszeit gravierende Funktionalitätseinbrüche zu erleiden oder sogar das Zerbrechen des Verbundes zu riskieren. 
Erster und zweiter Punkt spielen hier eng zusammen. Kaum war das Ziel eines wirklich integrierten Bibliothekssystems mit Aleph zum Greifen nahe, kamen die neuen Anforderungen an die Verwaltung elektronischer Ressourcen und deutlich besserer Statistik- und Analysefähigkeiten. Dass die in Aleph verwendete Architektur und Softwaretechnik nach dieser langen Lebenszeit nicht mehr so ganz zeitgemäß dasteht, sieht jeder Systembetreiber. Als die Herstellerfirma Ex Libris auf den internationalen Benutzertreffen über ein Nachfolgesystem konkreter zu sprechen begann, war zumindest mir klar, dass wir uns mit der Thematik beschäftigen müssen. Hier war ein umfassender Meinungsbildungs- und Selbstorganisationsprozess erforderlich, denn ein weisungsbefugtes Ministerium mit einem zentralen Budgettopf für solche Unternehmungen im Bibliotheksbereich gibt es ja nicht mehr. Nach intensiver Diskussion und mit Wohlwollen der Verbundvollversammlung hat sich dann eine gewichtige Pioniergruppe aus 14 Einrichtungen zusammengefunden, die das Abenteuer der glücklicherweise erfolgreich verlaufenen Ausschreibung und der Erstimplementierung auf sich genommen hat. Ich glaube, dass wir - auch mit unserer exzellenten externen juristischen Unterstützung - für alle anfänglichen Herausforderungen vorzeigbare Lösungen gefunden haben. Für unsere internen Abläufe Selbstverpflichtungen. Für die gemeinsamen Verhandlungen das wechselseitige Vertrauen, basierend auf unserer langjährigen Zusammenarbeit und die intensiven Gespräche untereinander. Eine geschickt formulierte Ausschreibung, um auch die verbliebenen Verbundteilnehmerlnnen später ohne Mehrklassengesellschaft in das neue Boot holen zu können. Eine Phase zur detaillierten Ausarbeitung grundsätzlicher Konzepte. Und einen Parallelbetrieb zwischen alter und neuer Systemumgebung, um den Mitarbeiterinnen und Mitarbeitern die gleichzeitige Arbeit in zwei unterschiedlichen Systemen zu ersparen. Leider ist es bei einem solchen Systemwechsel anscheinend nie eine besondere, sondern eine standardmäßige Herausforderung, dass alle Kräfte weit über jedes normale Maß beansprucht werden.

\section{Positive Aspekte des Systemwechsels}

Bauer: Bisher haben 13 Einrichtungen und der Verbund den Umstieg von Aleph zи Alma vollzogen. Vorangegangen sind ein zweijähriges Vergabeverfahren sowie eine Konzeptions-, eine Vorbereitungs- und eine Implementierungsphase. Wenn Du auf die vergangenen Jahre zurückblickst: Was ist aus Deiner Sicht bei der Umstellung von Aleph zu Alma gut gelaufen? 
Hamedinger: Die Zusammenarbeit der 14 Auftraggeber (erweitert um Vertreter der IT-Dienste) und auch weiterer Verbundteilnehmerlnnen bei der Vorbereitung der Ausschreibung und der Verhandlungsführung bis zum Zuschlag ist sehr gut gelaufen. Ebenso waren schlussendlich die Anstrengungen der Fachexpertinnen und -experten, aller koordinierenden Personen und aller Involvierten erfolgreich; wirklich gut gelaufen wäre es, wenn die Wanderung durch das Tal der Tränen etwas weniger lang gewesen wäre.

Wir haben die Zeitpläne eingehalten, auch wenn das manchmal ein Ritt über den Bodensee und jede vorgesehene Pufferzeit verbraucht war.

Es kam zu einer Wissensverbreiterung im Verbund, viele bisher nicht in dieser Form eingebundene und insbesondere jüngere Personen haben sich eingebracht, spezifische Kooperationsformen entwickelt und stellen mit dem erworbenen Wissen jetzt eine neue Basis für den Bibliotheksbetrieb dar.

Durch viel konzeptionellen Fleiß und Überzeugungsarbeit konnten wir die Implementierung der GND in einer verwendbaren Form erreichen. Die Zusammenarbeit mit Ex Libris war und ist in vielen Bereichen sehr gut, wenn auch nicht in allen; dort findet man exzellente Leute.

Der Parallelbetrieb mit dem bidirektionalen MAB2-MARC21-Konverter funktioniert zufriedenstellend.

Auch wenn ich damit sicher Widerspruch herausfordere, bin ich auf Grund vieler Aspekte inzwischen noch mehr als früher davon überzeugt, dass wir bei allen Problemen mit dem neuen System einen guten Zeitpunkt für die Systemumstellung erwischt haben.

\section{Negative Aspekte des Systemwechsels}

Bauer: Bei Projekten jeglicher Art ist man nach deren Abschluss zumeist schlauer, sodass sich die Frage stellt: Welche Dinge würdest Du bei der Umstellung von Aleph zu Alma im Rückblick anders machen?

Hamedinger: Sarkastisch gesprochen wäre der frische Erkenntnisgewinn doch glatt ein Grund, sofort wieder so ein Projekt anzugehen ...

Das ist insofern eine schwierige Frage, da wir manches, was wir anders machen sollten, wahrscheinlich gar nicht anders machen könnten. Damit meine ich vor allem die personelle Situation in den Bibliotheken, aber auch in der Verbundzentrale. Es hat sich im Lauf des Projekts die altbekannte Tatsache bestätigt, dass in unserem Metier besonders für die Systemumstellung speziell qualifiziertes Personal erforderlich ist, dieses sich nicht einmal theoretisch vermehren lässt, das vorhandene daher neben dem Regel- 
betrieb das neue System einrichten muss und insbesondere besonders heikle Aufgaben immer an denselben Personen hängen. Nur in wenigen Fällen haben hier organisatorische Maßnahmen etwas Linderung gebracht.

Ansonsten fallen mir folgende Änderungen ein:

Ein a priori realistischerer Kostenansatz für die Vorbereitung und Durchführung des Vergabeverfahrens.

Bessere Einschätzung der Komplexität des Formatkonverters zwischen Aleph und Alma in beide Richtungen und deshalb rechtzeitige Abtrennung als separates Projekt.

Frühere Einbindung weiterer Betroffener, insbesondere universitärer IT-Bereiche, zur Vermeidung unerquicklicher Diskussionen und atmosphärischer Störungen.

Bessere Absprache mit den anderen Auftraggebern, wie weit für die Umstellung in den Häusern Prioritäten verschoben und die unbedingt nötigen Personen anderweitig entlastet werden können.

Einrichtung nicht nur einer Projektkoordination an der Verbundzentrale, sondern eines zusätzlichen zentralen Projektmanagements von zwei bis drei Personen als Gegenüber der Lieferfirma und zur Unterstützung der einzelnen Bibliotheken. Allerdings wäre die Finanzierung durch die Verbundzentrale allein nicht möglich gewesen, sondern die weiteren Auftraggeber hätten diese Kosten übernehmen müssen. Bei den Gesamtkosten der Migration sollte ein solcher Posten bei rechtzeitiger Absprache wohl unterzubringen gewesen sein.

Last but not least würde ich über die Form des Parallelbetriebs nochmals nachdenken, auch wenn ich nicht an eine grundsätzlich andere Möglichkeit als die schließlich dafür gewählte glaube.

\section{Parallelbetrieb und Abschluss der Umstellungsphase}

Bauer: Der Österreichische Bibliothekenverbund besteht aus 91 Einzelinstitutionen, von denen bisher 14 auf das neue Bibliothekssystem umgestiegen sind. Die überwiegende Mehrzahl der Institutionen lebt noch in der Aleph/Alephino-Welt. Wie lange wird der Parallelbetrieb laufen? In welchem Zeitrahmen werden die weiteren Verbundbibliotheken die Aleph/Alma-Umstellung vollziehen?

Hamedinger: Der Parallelbetrieb wurde eingerichtet, weil es mit den vorhandenen Ressourcen unmöglich ist, alle verbliebenen Verbundteilnehmer Innen mit einem Schlag in den neuen Verbundkern zu überführen, insbesondere wegen der Besonderheiten und organisatorischen Rahmenbedin- 
gungen der einzelnen Einrichtungen. Der Umstieg erfolgt in Gruppen verkraftbarer Größe in halbjährlichen Intervallen, die von Ex Libris inzwischen „Waves“ genannt werden. Nach den marschierenden Kohorten der Pioniergruppe, die die Bahn gebrochen haben, wogen und wallen die Nachfolger jetzt zum neuen Verbundkern und gliedern sich dort ein. Anfang September ist eine erste Sondergruppe von drei Bibliotheken recht problemlos in Betrieb gegangen, wir sind jetzt also schon 17. Die nächste Wave hat mit der Umstellung begonnen und wird Anfang 2019 die Produktion aufnehmen. Die letzte Wave steigt plangemäß im September 2021 um, zeitnah wird danach auch das zentrale Aleph-System abgeschaltet, das während des Parallelbetriebs ja immer noch erhebliche Ressourcen bindet. Zumindest für die Verbundzentrale ist Aleph dann nach fast 23 Jahren Geschichte.

Die Besetzung der Waves ist inzwischen recht weit fortgeschritten, es bestehen nur mehr kleine Unsicherheiten. Die Umstellung ist allerdings nicht bei allen Einrichtungen beschlossene Sache. So scheidet eine Einrichtung aus dem Verbund aus, eine will Aleph behalten und vier haben noch keine Entscheidung getroffen.

\section{Weitere Geschäftsfelder der OBVSG}

Bauer: Die OBVSG widmet sich neben der Kernaufgabe als Verbundzentrale weiteren Geschäftsfeldern. Welche Services werden neben dem Themenkomplex Aleph/ Alma derzeit von der OBVSG betrieben?

Hamedinger: Zumindest nach meinem Verständnis gelten einige Kernaufgaben der Verbundzentrale für alle technischen Aktivitäten und werden daher auch überall wahrgenommen. Die Integrität unserer Daten ist ein wesentliches Ziel. Datenexpertise und erforderlichenfalls automatische Datenkorrekturen sind daher im Hintergrund immer dabei. Auch die bestehende AuBenvertretung in den verschiedenen Gremien, Zusammenarbeit mit Partnerverbünden, Mitwirkung bei der Standardisierung insbesondere im deutschen Sprachraum, aber auch darüber hinaus, durch die Größe des Verbundes unterfütterte Kontakte mit Herstellern und Lieferanten wirken schlussendlich auf die Qualität und Möglichkeiten der einzelnen aktiven Dienste ein.

Bevor ich verschiedene Dienste einfach aufzähle, möchte ich ein bisschen ausholen und unsere grundsätzliche Philosophie dazu etwas erläutern. Es wird nicht so gern gehört, aber jeder Dienst verursacht natürlich Kosten, die irgendwer tragen muss. Der Bund lässt bei neueren zentralen Diensten seit Jahren aus und zusätzliche Dienste sind laut gesetzlicher An- 
ordnung jedenfalls kostendeckend zu erbringen. Wir müssen daher immer überlegen, wie wir den Übergang von einem Projekt, das üblicherweise halbwegs problemlos finanziert werden kann, meistens in gerade Aufmerksamkeit auf sich ziehenden neuen Bereichen stattfindet und daher "sexy“ ist, zu einem regulären und stabil laufenden Dienst hinbekommen, der dann natürlich mit den aufregenden neuen Problemen, auf die dann später das Interesse gerichtet ist, meistens nicht mehr soviel zu tun hat. Etwas, das eben einfach funktioniert und daher nicht mehr besonders beachtet wird - erst wieder, wenn es Probleme gibt. Deswegen konzipieren wir solche Dienste im Regelfall so, dass sie immer für eine möglichst große Gruppe interessant und verwendbar sind, damit sich die Basiskosten verteilen und dass sie in das Gesamtbiotop der bisherigen Systemumgebung passen. Nur wenn es uns gelingt, dadurch Vorteile für alle Nutzer des jeweiligen Dienstes zu erzeugen, kommen wir zu einem robusten und finanzierbaren Dienst.

Im Lauf der Jahre wurden neben den Integrationsverfahren zur Hauptanwendung Aleph bzw. nun Alma mehrere Dienste aufgebaut. In chronologischer Reihenfolge sind beispielhaft folgende Dienste aktiv: Aleph-Sharing, das die Nutzung eines Aleph-Systems durch mehrere kleinere Einrichtungen erlaubt und diesen damit auch die Eingliederung in eine homogene Verbundarchitektur ermöglicht - übrigens ein Umstand, den ich strategisch gesehen sehr hoch bewerte; die Anbindung von Aleph und Alma an SAP über die Middleware an der Bundesrechenzentrum $\mathrm{GmbH}$, mit einer Ausnahme; die Versorgung mit sfx und/oder MetaLib; die Einführung von Suchmaschinentechnologie in Form von Primo und der Ersatz der vorherigen OPAC-Technologie; die Bereitstellung von Repositorien auf der Basis von Visual Library; der URN-Dienst zur permanenten Identifikation von elektronischen Dokumenten. Die leidige Unterbrechung bei der Bestandsdatenlieferung an die ZDB wird nun hoffentlich auch bald beendet sein.

Darüber hinaus ist die OBVSG offizielle ISIL-Agentur für Österreich, betreibt ohne Anspruch auf Vollständigkeit eine Adressdatenbank der wissenschaftlichen Bibliotheken in Österreich samt Sigel- und ISIL-Verzeichnis und versucht die Verbundstruktur hinreichend zu dokumentieren, da davon die reibungslose Funktion vieler Dienste abhängt.

\section{Kooperation E-Medien Österreich}

Bauer: 2008 übersiedelte die Geschäftsstelle der 2005 auf Initiative des Forums Universitätsbibliotheken Österreichs gegründeten Kooperation E-Medien Österreich 
(KEMÖ) von der Universität Graz an die Österreichische Bibliothekenverbund und Service GmbH nach Wien. Welche Motive waren für Dich für diese Transferierung, die Du sehr stark unterstützt hast, ausschlaggebend? Welche Vorteile siehst du in der Unterbringung der KEMÖ unter einem gemeinsamen Dach mit der OBVSG?

Hamedinger: Die KEMÖ ist eine sehr erfolgreiche Unternehmung, die zwar auf Initiative des ubifo entstanden ist, sich aber besonders durch die Fachkompetenz der entsandten Vertreterinnen und Vertreter und die richtige zentrale Koordination gut entwickelt hat. Die KEMÖ ist von den Mitgliedern her zwar nicht deckungsgleich mit dem Verbund, die Überschneidung ist allerdings sehr groß. Sie ist durch den Teilnahmevertrag sehr basisdemokratisch organisiert, was viele Vorteile, aber auch einige Herausforderungen mit sich bringt und unbedingt eine handlungsfähige zentrale Stelle erfordert. Als die Idee einer Ansiedlung der nunmehrigen Geschäftsstelle an der OBVSG herangetragen wurde, habe ich das vorbehaltlich einer ordentlichen Regelung natürlich unterstützt und das aus mehreren Gründen. Die KEMÖ selbst ist keine Rechtsperson, aber die Geschäftsstelle muss irgendwo organisatorisch untergebracht sein, schließlich müssen Arbeitsverträge geschlossen und Gehälter bezahlt werden. Eine Einbindung in einen Produkte beziehenden KEMÖ-Teilnehmerlnnen kann immer zu Interessenkollisionen führen oder zumindest den Anschein solcher erwecken. Außerdem ist eine solche Geschäftsstelle in der Organisation einer Universität eher weit weg von Personalverwaltung und Rektorat, sodass eventuell nötige schnelle Maßnahmen nicht leicht zustande kommen. All diese vielleicht bürokratisch wirkenden Überlegungen waren neben den Tatsachen, dass die elektronischen Ressourcen immer wichtiger werden und die OBVSG der letzte gesetzliche Träger des Verbundgedankens ist, maßgeblich für mein Interesse der Ansiedlung der Geschäftsstelle an der OBVSG. Sie ist inzwischen als eigene Abteilung eingerichtet und hat unmittelbaren Kontakt zur Geschäftsführung. Damals gab es einige Befürchtungen zu Kontrollverlust durch die Mitglieder und einen Machtzuwachs der OBVSG, konkret meiner Person. Aber ich glaube, dass wir die Turbulenzen der letzten Jahre gerade durch die gewählte Zusammenarbeit zwischen OBVSG und KEMÖ, insbesondere dem Kooperationsausschuss, bewältigen konnten und nun endlich in ruhigem Fahrwasser angelangt sind. Den Normalbetrieb stimmen Geschäftsstelle und Kooperationsausschuss im Rahmen der verfügbaren Ressourcen selbst $a b$ und nur bei unerwarteten bzw. Notfällen greife ich ein, möglichst in Abstimmung mit dem Kooperationsausschuss. Die kurzen Wege und vor allem das Verständnis für die fachlichen Erfordernisse halte ich 
dabei für die größten Vorteile. Ich muss inzwischen nur mehr sehr selten Feuerwehr spielen und hoffe, dass das auch so bleibt und gegebenenfalls so zufriedenstellend wie bisher gelingt. Insgesamt haben wir mit einigen Mühen gemeinsam eine gute Lösung aus Selbstverwaltung und Notfallnetz gefunden, die wir für jede neue Vertragsperiode weiterentwickeln. Als ein Zeichen dieser Zusammenarbeit ist auch die OBVSG selbst seit 2015 Mitglied in der KEMÖ, allerdings ohne Bezug von Produkten und somit weiterhin in einer neutralen Position.

\section{Finanzierung der OBVSG}

Bauer: Ein wichtiges Thema für jede Firma - und somit natürlich auch für die OBVSG als Firma mit einer 100\%igen Bundesbeteiligung - ist deren Finanzierung. Die OBVSG erhält seit deren Ausgliederung im Jahre 2002 einen gedeckelten Jahreszuschuss des Bundes in Höhe von 1,7 Mio. Euro. Wie ist es möglich, unter diesen finanziellen Voraussetzungen als Firma zu bestehen? Wie würde für Dich eine optimale Finanzierung der OBVSG aussehen, damit sie auch in Zukunft ihren Auftrag als Verbundzentrale leisten kann?

Hamedinger: Das ist leider tatsächlich ein Thema, das mir immer mehr Kopfzerbrechen bereitet. Der Bund hat uns mit einem grundsätzlich definierten Aufgabenspektrum, einer Basisdotation für umschriebene Dienste und dem Auftrag, alle darüber hinausgehenden Dienste zumindest kostendeckend zu erbringen, am 1. Jänner 2002 in die Selbständigkeit entlassen. Diese Basisdotation von ganz genau 1,72 Millionen Euro wurde bisher um keinen Cent erhöht, weder durch Gesetz noch durch fallweise Sonderdotationen. Die Intention, die OBVSG schlagkräftig und unabhängig von ministeriellen Entscheidungsabläufen und kameralistischen Zwängen zu machen, wurde erreicht und ich halte diese Entscheidung für gut. Die Intention des Finanzministeriums "gehet hin mit eurem Obolus und schlagt euch durch, wir fühlen uns finanziell nicht mehr zuständig" wurde leider auch erreicht. Hintergedanke war natürlich, zu besserer Organisation, Beseitigung von Leerläufen und der Hebung von Synergien zu zwingen. Allerdings kosten Optimierungsmaßnahmen irgendwann mehr als sie einbringen und wenn, bildlich gesprochen, der Fettrand weggeschnitten ist, geht es ins Fleisch. Nebenbei bemerkt war dieser Fettrand bei uns immer schon sehr dünn und im Verwaltungsbereich gar nicht vorhanden.

Um die Dimensionen zu erläutern: Der Verbraucherpreisindex hat sich seit der Ausgliederung der OBVSG um über 36 Prozent erhöht, das ent- 
spricht jährlich dem erheblichen Betrag von 620.000 Euro. Die Gehaltssteigerung im ADV-Schema des Bundes liegt bei etwa 55 Prozent, aktive Beamte haben wir derzeit keine, ich selbst als das letzte Fossil dieser Art wäre inzwischen um über 100 Prozent „teurer". Trotzdem konnten wir in der ganzen Zeit mit Ausnahme des Jahres der Primoeinführung positiv bilanzieren. Gelungen ist uns das aus mehreren Gründen: Seit Anbeginn entwickeln wir sinnvolle integrierende Dienste, deren Einnahmen ganz wesentlich zum Budget der OBVSG beitragen. Die Kosten für Hardware steigen deutlich moderater als die benötigte Leistung. Die Personaldecke ist sehr dünn, Vertretungen für Spezialisten gibt es kaum. Neueinsteiger fangen nicht in den höchsten Gehaltsrängen an und können mit Bundesschemata inklusive Biennalsprüngen und entsprechenden Einstufungen nicht mehr rechnen. Last but not least das Brennen für die Sache und der hohe Grad an Selbstausbeutung, etwas, was in Bibliotheken auch nicht unbekannt ist.

Das ist insgesamt eine bedenkliche Situation, denn wenn wie jetzt im Zuge der Alma-Umstellung eines dieser finanziellen Arbeitspferde wegbricht, ändern sich die Gegebenheiten sehr schnell ins Negative. Sowohl Aufsichtsrat als auch Eigentümer sind sich der Problematik bewusst, aber leider geht es insgesamt um zu kleine Beträge für die höhere Politik.

Nun aber zum Wunschkonzert bei der Finanzierung: Die grundsätzliche Aufteilung in vom Bund über den Jahreszuschuss finanzierte Grunddienste und kostenpflichtige Zusatzdienste hat sich bewährt und sollte beibehalten werden. Allerdings ist eine Aufgabenkritik für die Grunddienste erforderlich, verbunden mit einer Neubewertung der erforderlichen Aufwendungen. Einzurechnen wären dabei jedenfalls auch die durch laufende Entbürokratisierungsmaßnahmen immer wieder verursachten Negativeinsparungen wegen der erforderlichen Bedienung von unter anderem Medientransparenzgesetz, Public Corporate Governance Kodex, Statistikerhebungen, Wirtschaftliche Eigentümer Registergesetz und gerade aktuell Datenschutzgrundverordnung. Im fachlichen Bereich wünsche ich mir, dass wir Aufgaben wie die Normdatenkoordinierung und erweiterte Metadatenkompetenz in den Katalog der zentralen Leistungen aufnehmen, dass Technologieverfolgung und Dienstentwicklung mit angemessenen Ressourcen berücksichtigt werden und dass wir weiterhin nicht für jeden Not- und Beratungsfall „die Hand aufhalten“ müssen und damit die Verbundzentrale als Problemanlaufstelle schwächen. Garniert mit regelmäBiger Evaluierung dieser Dienste und entsprechender Anpassung der Bundesabgeltung für die definierten zentralen Dienste unter Berücksichtigung vernünftiger Gehälter. 


\section{8. „Digitalisierung“ - Chance für die OBVSG?}

Bauer: Die öffentliche Diskussion ist derzeit vom Schlagwort „Digitalisierung“ geprägt. Welche Chancen bieten sich im digitalen Zeitalter für Bibliotheken? Gibt es in diesem Zusammenhang Themenfelder, die die OBVSG in den nächsten Jahren übernehmen wird?

Hamedinger: „Digitalisierung“ ist auch so ein Schlagwort, das je nach Situation in verschiedensten Bedeutungen verwendet wird. In unserem Metier haben wir die Umwandlung von analogen Materialien in digitale Abbilder und die Behandlung generisch erzeugter digitaler Informationsträger. Schlussendlich müssen die Ergebnisse vernünftig und wieder auffındbar verwaltet, möglichst barrierefrei weitgestreut zugänglich gemacht und langfristig verfügbar gehalten werden. Im Bereich der Retrodigitalisierung haben wir an der OBVSG eine Lösung im Portfolio, die Langzeitverfügbarkeit von Bibliotheksmaterialien ist weit genug entwickelt, dass wir über einen entsprechenden Dienst nachdenken können und das gerade in ziemlicher Gärung befindlichen Thema Forschungsdaten können wir neben dem Systemwechsel auf Alma nur als Zaungast verfolgen; mangels Ressourcen müssen wir erst einmal abwarten, wohin die Reise geht und ob überhaupt etwas herauskommt, was in unsere Dienstumgebung passen könnte.

\section{Charakteristika des Österreichischen Bibliothekenverbundes}

Bauer: Der Start des Österreichischen Bibliothekenverbundes erfolgte 1988, als der Online-Betrieb mit der Bibliotheksverwaltungssoftware BIBOS aufgenommen wurde. Somit blicken wir heuer auf 30 Jahre Leben im Verbund zurück. Du kennst aufgrund der gemeinsamen regelmäßig stattfindenden Treffen auch die anderen deutschsprachigen Verbünde sehr gut. Gibt es etwas, das den Österreichischen Bibliothekenverbund besonders auszeichnet?

Hamedinger: Eher schon 31 Jahre, denn die Implementierung lief 1987 an, aber es war tatsächlich der Start der großflächigen Zusammenarbeit, da der eigentlich erste Verbundkatalog, die Österreichische Zeitschriftendatenbank, zwar seit 1984 existierte, aber eben nur von einem kleinen Kreis von Spezialisten aus der Zeitschriftenbearbeitung bedient wurde.

Unsere herausragende Besonderheit, nämlich die sehr tiefgreifende und partnerschaftliche Zusammenarbeit zwischen Verbundzentrale, teilnehmenden Bibliotheken und auch zwischen den teilnehmenden Bibliotheken 
verdanken wir meiner Meinung nach einer Reihe von Umständen: der Zuständigkeit einer einzigen Fachabteilung eines einzigen Ministeriums zur rechten Zeit; den Problemen mit dem ersten System BIBOS; der Auswahl von Aleph 500 als Nachfolger mit der damit möglichen homogenen Verbundumgebung; der für ihre Bereiche jeweils gleichwertigen Lokalsysteme zum Zentralsystem und der gemeinsamen systemtechnischen Sprache. Und natürlich dem Fehlen von Geldquellen, wie es sie in Deutschland gibt, insbesondere der fehlenden Infrastrukturförderung durch die Forschungsförderungsorganisationen und den nicht vorhandenen Zentralmitteln.

Insgesamt haben diese Umstände zu einer Bündelung von Kenntnissen sowie viel Pragmatismus und Flexibilität geführt, die in einer durchaus „familiären Atmosphäre" mündet. Trotz der einen oder anderen Meinungsverschiedenheit stehen hinter allem gemeinsame Intentionen. Dieses Biotop ist nicht selbstverständlich und trägt am meisten zu unserer individuellen Note bei. Für die nachfolgende Generation sind das alles Selbstverständlichkeiten und deswegen hoffe ich sehr, dass wir davon nichts durch schleichende Geringschätzung verlieren. Zerstört ist bald etwas, aber aufgebaut nicht.

\section{Perspektive für Bibliotheken und Bibliotheksverbünde}

Bauer: Du warst vor Deiner Tätigkeit in der Verbundzentrale - von 1986 bis 1993 - auch Leiter der Fachbibliothek für Mathematik, Statistik und Informatik an der Universität Wien. Somit hast Du auch einen sehr guten Einblick, in welchem Umfeld wissenschaftliche Bibliothekarinnen und Bibliothekare agieren. Wenn Du nun zehn Jahre nach vorne blickst: Denkst Du, dass wissenschaftliche Einrichtungen dann noch Bibliotheken - und auch Bibliotheksverbünde - benötigen? Und wenn Du diese Antwort, wie ich hoffe, mit "Ja" beantwortest, welche Aufgaben werden sie zu erfüllen haben?

Hamedinger: Das ist jetzt also die Frage, mit deren Beantwortung man sich in zehn Jahren möglicherweise gehörig lächerlich macht. Obwohl mir die Gabe des Kristallkugellesens nicht gegeben ist, antworte ich in beiden Fällen mit ja, auch wenn ich über die konkrete Ausgestaltung nichts sagen kann. Ich habe mich zwar mit der ganzen Bandbreite bibliothekarischer Arbeiten an einer Fachbibliothek einer Universität beschäftigt, aber das ist, wie Du anfänglich schon festgestellt hast, inzwischen 25 Jahre her. In meiner damals fast siebenjährigen Tätigkeit hatte ich das Glück den Übergang vom Zettelkatalog zum elektronischen Bibliothekssystem mitzuma- 
chen und so die Umstellung in der Erschließung mit einzuleiten. Seither ist das aber alles selbstverständlich und es hat sich noch viel mehr in den Abläufen verändert, was ich jedoch nur mehr am Rande und aus einem anderen Blickwinkel mitbekommen habe. Das Universitätsorganisationsgesetz 1993, die Ablösung von BIBOS durch Aleph, die Entstehung der Fachhochschulen und besonders das Universitätsgesetz 2002 haben ihre Spuren hinterlassen und wirken auch in die Zukunft - allerdings nicht nur positiv.

In dem ganzen Informationsmeer muss es weiterhin stabile Ankerplätze geben, an denen man die Chance hat, zuverlässige von ungeprüfter Information zu scheiden, und wo diese zuverlässigen Informationen auch zugänglich sind - und zwar für breite Bevölkerungsschichten und auf Dauer. Es wird sich irgendwann doch die Erkenntnis Bahn brechen, dass dahinter auch eine gewisse dauerhafte Strategie liegen sollte und nicht nur in der momentanen Kurzatmigkeit an die nächsten zwei Jahre gedacht wird. Du selbst leitest ja eine Bibliothek in einem Bereich, wo man immer wieder hört, dass alles was vor mehr als fünf Jahren publiziert wurde, obsolet sei. Das mag vielleicht die Meinung mancher Forscher sein, wir sind zentral auch für diese da, aber - und das scheint mir inzwischen zu kurz zu kommen - nicht nur, sondern auch für zukünftige Generationen. Neben den Aspekten des Ordnens, Aufbewahrens und Bereitstellens ist vor allem die sachgerechte Beschaffung auch weiterhin die Aufgabe von Bibliotheken. Der Umgang mit Verlagen, insbesondere mit den Aspekten elektronischen Materials, ist eine ureigenste Tätigkeit von Bibliotheken. Es gehört mit zu ihren Aufgaben, dafür zu sorgen, dass die mit erheblichen Beträgen Steuergeld beschafften Informationen auch langfristig der finanzierenden Öffentlichkeit zur Verfügung stehen und sie damit deren Interessen vertreten. Die konkrete Ausgestaltung und technische Ausrüstung wage ich nicht vorherzusagen, aber dass wir sozusagen den Stafettenstab so gut wie möglich und neutral an die folgenden Generationen weiterreichen, halte ich für eine Notwendigkeit. Welche Erkenntnisse wie in die Zukunft wirken, kann niemand vorhersehen. Genauso wie man jetzt über viele Ansichten von vor zweihundert Jahren lacht, gab es damals Ideen, die noch heute nachwirken oder das Fundament für ganze Wissensgebäude abgeben. Auch unsere Zeit wird einmal ähnlich gesehen werden. Viele Ergebnisse sind nicht nachzuvollziehen, wenn man die Ideengeschichte nicht kennt. Schon aus ideellen Gründen und vielleicht naiv glaube ich, dass Bibliotheken ein wichtiger Baustein von Gesellschaften mit mündigen Mitgliedern sind. In einer solchen möchte hoffentlich nicht nur ich leben, also erklärt sich dieser Teil meiner positiven Antwort. 
Viel handfester sind die Vorteile, die sich aus Zusammenschlüssen in Verbünden oder verbundähnlichen Gebilden wie der KEMÖ ergeben. Wir sehen sowohl bei den Verlagen als auch bei den Systemlieferanten massive Konzentrationsprozesse. Mit ganz wenigen Ausnahmen hat eine einzelne Bibliothek weder die Marktmacht noch die Ressourcen, um mit diesen Firmen auf Augenhöhe verhandeln zu können. Auf der Ebene eines Zusammenschlusses bestehen dafür zumindest realistische Chancen, wie sich sowohl bei Pionierprojekten in der Literaturbeschaffung als auch bei der Ablöse von Aleph gezeigt hat. Solange wir auch dafür sorgen, dass unsere Daten ausreichende Qualität haben und dass damit gearbeitet wird, können die durch einen Verbund möglichen Synergieeffekte auch genutzt werden. Pragmatismus und Flexibilität sind hier die Schlüssel. Solange es in den Grundsätzen ein gemeinsames Verständnis unter den Teilnehmerlnnen gibt, wird der Verbund Vorteile für alle bringen. Auch wenn sich die Schwerpunkte innerhalb des Verbundes verschieben können, erwarte ich für die nächsten 10 Jahre keine wirkliche Vereinfachung im Gesamtaufgabenfeld der Bibliotheken und somit gehe ich auch weiterhin von einer Existenzberechtigung für Bibliotheken und Verbünden in diesem hochkomplexen Umfeld aus.

\section{Wolfgang Hamedinger}

Mag. Wolfgang Hamedinger, Jahrgang 1960, studierte Mathematik und Physik an der Universität Wien, wo er 1984 das Diplomstudium Mathematik abschloss. Nach einer Zeit als Vertragsassistent und Lektor am Institut für Mathematik der Universität Wien trat er 1986 in den Bibliotheksdienst ein und übernahm die Leitung der dortigen Fachbibliothek für Mathematik, Statistik und Informatik. Im Jahr 1993 wurde er zum Leiter der Planungsstelle für wissenschaftliches Bibliothekswesen an der Österreichischen Nationalbibliothek bestellt, deren Nachfolgeorganisation Arbeitsgruppe Bibliotheksautomation im Bundesministerium für Bildung, Wissenschaft und Kultur er ab 1996 vorstand. Seit der mit dem Jahr 2002 erfolgten Ausgliederung der Arbeitsgruppe als Österreichische Bibliothekenverbund und Service $\mathrm{GmbH}$ ist er deren Geschäftsführer und damit auch operativer Leiter des Österreichischen Bibliothekenverbundes. 


\section{Publikationen (in Auswahl):}

Von der ÖZDB zur ZDB: Ein Zwischenbericht. In: Mitteilungen der Vereinigung Österreichischer Bibliothekarinnen \& Bibliothekare 61 (2008), H. 1, S. 45-51. Online: http://hdl.handle.net/10760/11329 10 Jahre Verbundorganisation neu: Verbundfamilie, Verbundarbeit, Kooperation. In: Die neue Bibliothek. Anspruch und Wirklichkeit. 31. Österreichischer Bibliothekartag, Innsbruck 2011. Graz / Feldkirch: Neugebauer, 2012, S. 173-178. Online: http://hdl.handle. net $/ 10760 / 23044$

Aktuelle und künftige Verbundentwicklungen: Einheit in der Vielheit. In: Die neue Bibliothek. Anspruch und Wirklichkeit. 31. Österreichischer Bibliothekartag, Innsbruck 2011. Graz / Feldkirch: Neugebauer, 2012, S. 179-196. Online: http://hdl.handle.net/10760/23045

Politik(absenz) - Strategie - Visionen: Vom Prinzip Hoffnung. In: Mitteilungen der Vereinigung Österreichischer Bibliothekarinnen \& Bibliothekare 65 (2012), H. 3/4, S. 388-393. Online: http://hdl.handle. net/10760/18462

Der Österreichische Bibliothekenverbund als Partner für die Universitätsbibliotheken. In: Universitätsbibliotheken im Fokus. Aufgaben und Perspektiven der Universitätsbibliotheken an öffentlichen Universitäten in Österreich, hrsg. von Bruno Bauer, Christian Gumpenberger und Robert Schiller. Graz / Feldkirch: Neugebauer, 2013, S. 248-256. Online: http://hdl.handle.net/10760/24296

Austrian Library Network and Next Generation Library System: Alma. In: Bibliothek. Forschung und Praxis 40 (2016), H. 3, S. 341-346. https://doi.org/10.1515/bfp-2016-0055

Mag. Bruno Bauer

ORCID: http://orcid.org/0000-0002-4729-331X Medizinische Universität Wien, Universitätsbibliothek E-Mail: bruno.bauer@meduniwien.ac.at

Mag. Wolfgang Hamedinger Die Österreichische Bibliothekenverbund und Service Gesellschaft m.b.H. E-Mail: wolfgang.hamedinger@obvsg.at 